\title{
IMPLANTATION OF A HEMODIALYSIS CATHETER DIRECTLY IN THE RIGHT ATRIUM DUE TO EXHAUSTION OF VASCULAR ACCESS
}

\author{
Pavlin Manoilov, Plamen Panayotov', Veselin Petrov², Georgi Todorov ${ }^{3}$, Milen Slavov ${ }^{1}$ \\ ${ }^{1}$ Department of Cardiac Surgery, St. Marina University Hospital, Varna \\ ${ }^{2}$ Department of Vascular Surgery, St. Marina University Hospital, Varna \\ ${ }^{3}$ Department of Imaging Diagnostics, St. Marina University Hospital, Varna
}

\begin{abstract}
INTRODUCTION: Patients on permanent hemodialysis need constant and secure vascular access with optimal flow to perform the procedure. Failure to provide such access can lead to life-threatening deterioration of the patient's condition.

CASE PRESENTATION: We report a case of implantation of a hemodialysis catheter directly in the right atrium (RA) in an emergency in a 46 year-old woman, placed on permanent hemodialysis (HD) for nine years due to end-stage renal disease with exhausted opportunities for vascular access over the years and unsuitable for peritoneal dialysis.

After a consultation between a cardiac surgeon, a vascular surgeon and an interventional radiologist, a decision was made to directly implant a permanent catheter for HD in the superior vena cava (SVC).

The operation was performed as a matter of urgency, operative access was through ministerotomy and the catheter was implanted directly in the right atrium (RA) due to thrombosis and fibrosis of the SVC. No cardiac complications associated with the procedure were observed in the postoperative period. On the first postoperative day, HD was performed, followed by others according to the therapeutic plan. The patient was discharged from the Cardiac Surgery Department on the 7th postoperative day.

CONCLUSION: The use of open surgical access and the implantation of a permanent HD catheter directly into the RA can be considered a life-saving procedure in HD patients with acute uremic syndrome and exhausted vascular access.
\end{abstract}

Keywords: hemodialysis, cardiac surgery, ministerotomy, exhausted vascular access

Address for correspondence:

Pavlin Manoilov

Department of Cardiac Surgery

St. Marina University Hospital

1 Hristo Smirnenski Blvd

9010 Varna

e-mail:manoilovp@gmail.com

Received: August 25, 2020

Accepted: September 19, 2020

\section{INTRODUCTION}

Georg Haas (1886-1971) performed the first human dialysis in the German city of Giessen in 1924. It was not until 1945 that Willem Johann Kolff, working in extremely difficult military conditions in the Netherlands, achieved the first clinically successful hemodialysis in a patient (1). 
Pavlin Manoilov, Plamen Panayotov, Veselin Petrov et al.

Clinical case: We present the case of a forty-sixyear-old woman with chronic renal failure (CRF) terminal stage, chronic glomerulonephritis, symptomatic hypertension, on HD treatment since 2011. Table 1 shows the vascular accesses performed in our hospital during the hemodialysis period. gent hemodialysis treatment due to exacerbation of uremic syndrome.

After a consultation between a vascular surgeon, a cardiac surgeon and an interventional radiologist with experience in the management of this type

Table 1. Clinical history

\begin{tabular}{|l|l|}
\hline Year & VASCULAR ACCESS \\
\hline 2011 & Left radiocephalic AVF \\
\hline 2012 & Left brachiocephalic AVF \\
\hline \hline 2013 & $\begin{array}{l}\text { AVF radiocephalic upper right antebrachium (18.05.2020) } \\
\text { Temporary catheter in v.jugularis int.dex. (30.05.2020) }\end{array}$ \\
\hline 2016 & $\begin{array}{l}\text { Extraction of the temporary jugular catheter. } \\
\text { Several control phlebography, establishing thrombosis of both iliac veins and internal jugular vein after } \\
\text { implantation of temporary catheters in the years. }\end{array}$ \\
\hline 2017 & Permanent catheter implantation in inferior cava vein by retroperitoneal approach. \\
\hline 2018 & Permanent catheter in inferior cava vein after phlebographic data for 50\% recanalization of left iliac vein. \\
\hline 2019 & Permanent catheter in left internal jugular vein. \\
\hline 2020 & $\begin{array}{l}\text { Replacement of the permanent catheter in the left internal jugular vein. } \\
\text { Permanent catheter placement in left subclavian vein. }\end{array}$ \\
\hline
\end{tabular}

In 2020 the patient was re-hospitalized in our hospital with a permanent tunneled catheter (PTC) implanted in the left subclavian vein. The procedure was performed in another medical institution, no medical documentation was available. The catheter was inserted in the area above the left breast, the surrounding skin was red and warm, the breast was tense and painful on palpation. The available PTC was impassable. Studies have shown the need for ur-

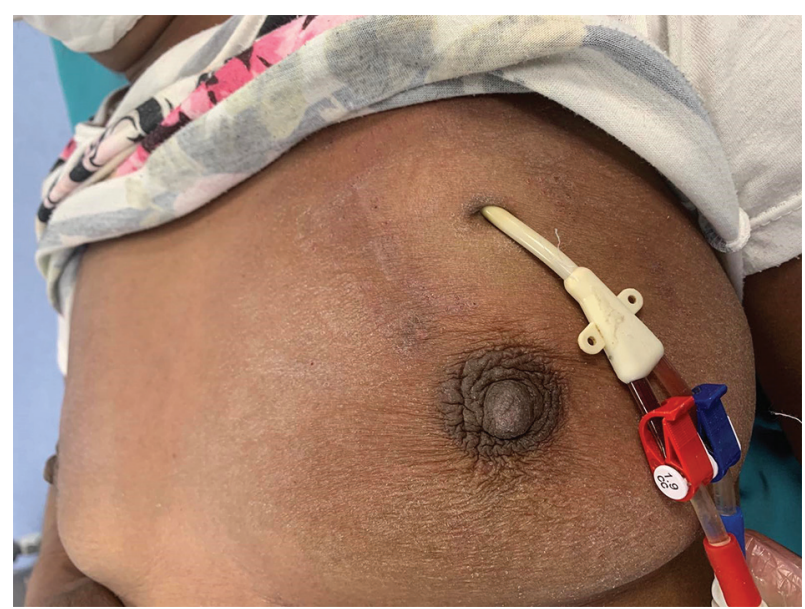

Fig. 1. Preoperative local status

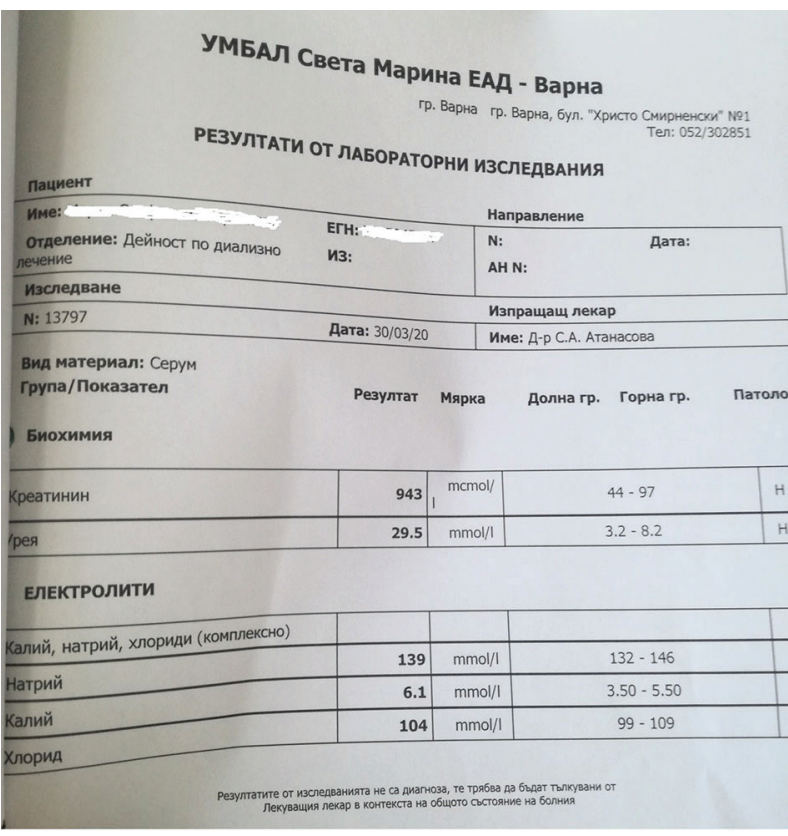

Fig. 2. Preoperative examination

of catheters, a decision was made for open surgical access to the superior vena cava (SVC).

Surgical access was hampered by the presence of multiple collateral veins in the area of the upper thorax, blood-filled, with a significant flow, easily 


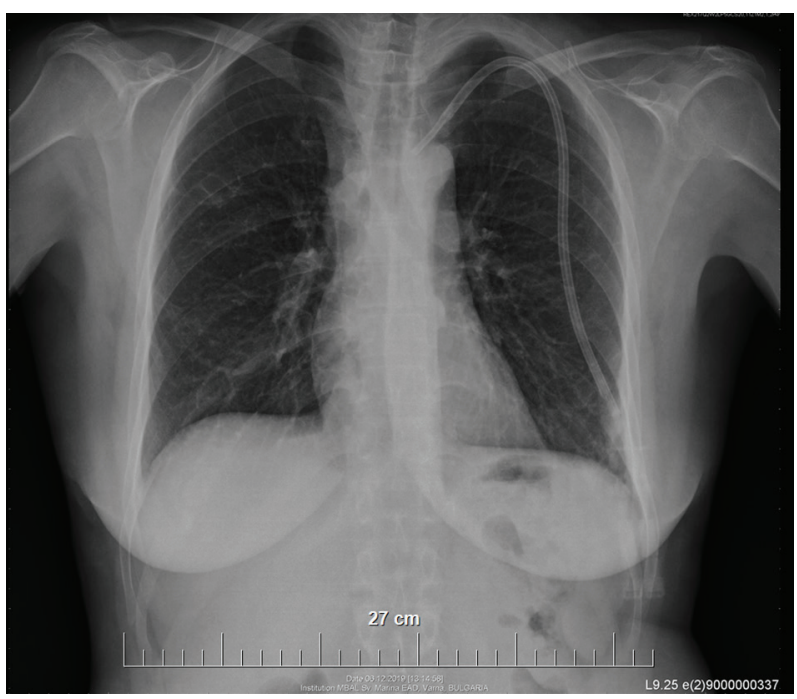

Fig. 3. Preoperative radiography

bleeding from injury, which necessitated the use of a cell-saver. Access to the anterior mediastinum was performed by partial upper sternotomy to the level of the 4 th rib on the right. Intense and thorough hemostasis due to the presence of multiple venous collaterals. A chronically inflammatory pericardium was

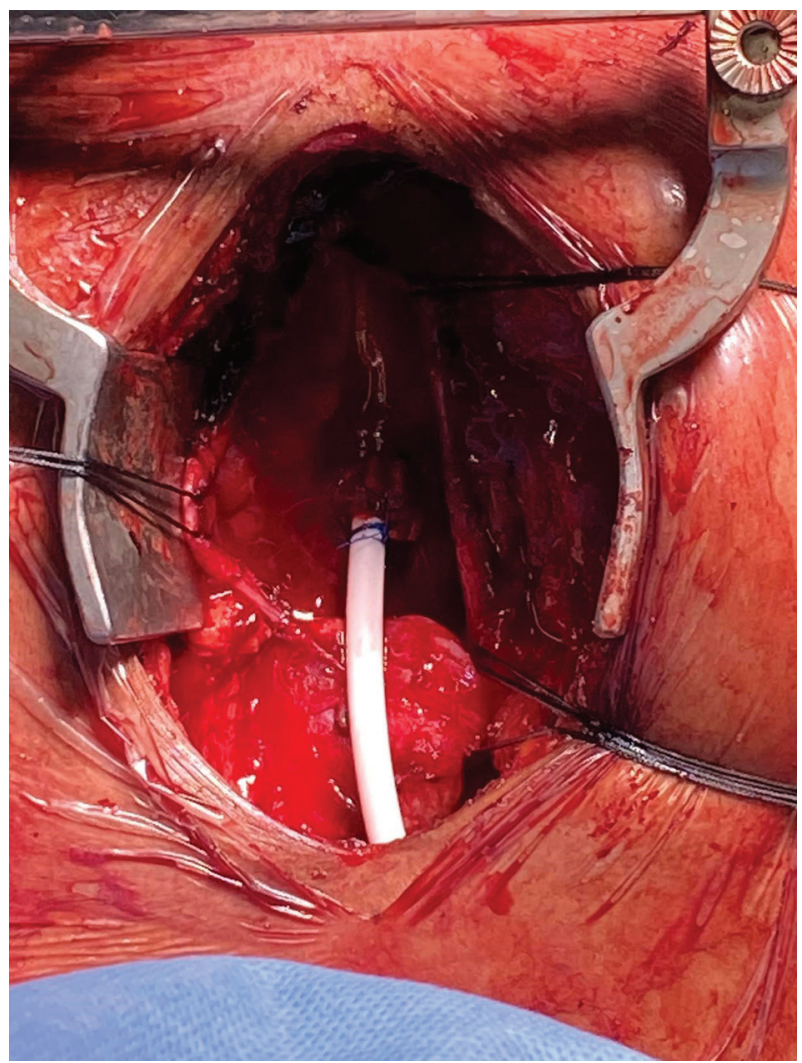

Fig. 4. Permanent catheter in the right ventricle found, strongly thickened, tightly fused with the underlying right ventricle (RV), as the type of constrictive pericarditis, with no clinical manifestations at present. A partial pericardiectomy was performed. During the exploitation of SVC, thrombosis was found with areas of fibrosis, which would prevent the introduction and proper functioning of the PTC. A decision was made to implant the catheter in the RA, which was done with two 4/0 polypropylene stitches reinforced with felt. The correct positioning of the two catheter tips in the RA and the inferior vena cava (IVC) was confirmed by intraoperative transesophageal echocardiography (TEE). The catheter was led supraclavicularly to the right through an established subcutaneous tunnel and stabilized according to the approved protocol. Its function was checked several times and was flushed with anticoagulant.

After closure of the ministernotomy and soft tissues, the non-functioning PTC was extracted from the left subclavian artery and surgical treatment of the field was performed to control the local infection.

The postoperative period was smooth. No adverse events related to catheter placement in the DP

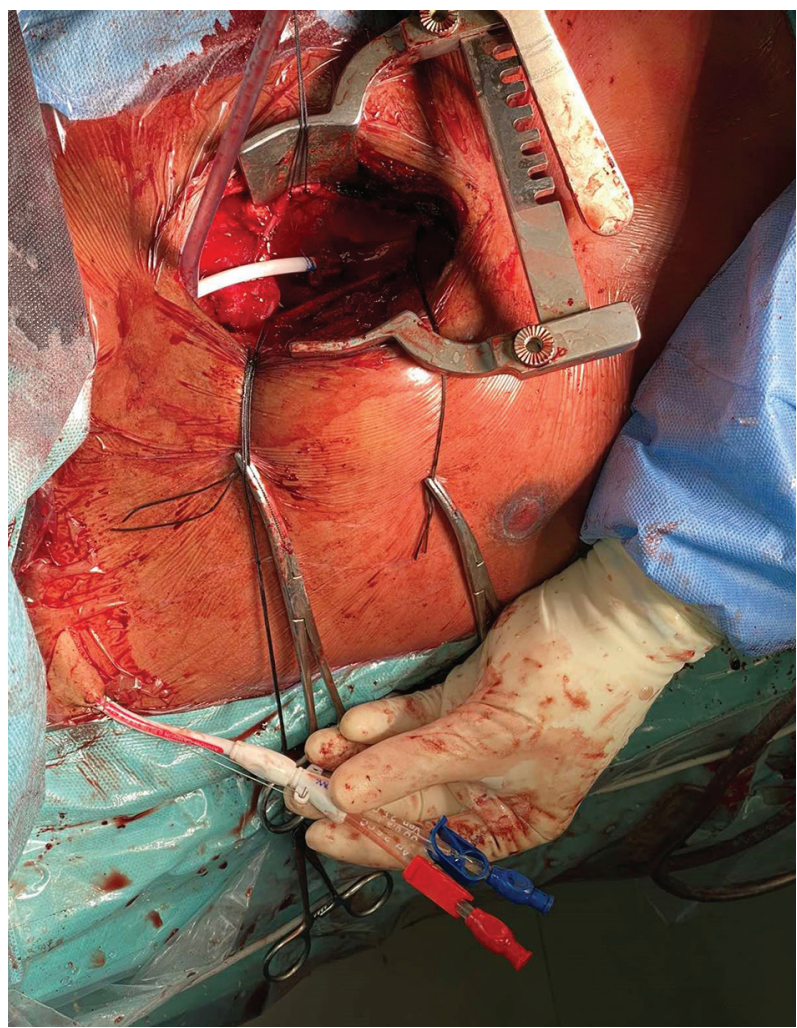

Fig. 5. Permanent catheter in the right supraclavicular area 
Pavlin Manoilov, Plamen Panayotov, Veselin Petrov et al.

were reported - arrhythmia, increase in cardiac enzymes, myocardial dysfunction. The antibiotic $(\mathrm{AB})$ therapy consisted of - vancomycin - $1.0 \mathrm{~g}$, with the next application being - after 4 days. On the first postoperative day, an HD was performed, followed by others according to the therapeutic plan. The infection in the area of the left breast was quickly overcome after treatment with appropriate antiseptics. The patient was discharged from the Cardiac Surgery Department on the 7 th postoperative day.

\section{DISCUSSION}

The number of patients with end-stage renal failure requiring HD is increasing every year worldwide. The need for permanent vascular access for such patients is essential to ensure adequate flow for hemodialysis sessions (2). The problem of providing vascular access in patients with persistent $\mathrm{HD}$ is one of the main reasons for the need for hospitalization and mortality. Thrombosis is the most common complication and the main cause of loss of vascular access $(3,4)$.

It is not common practice to place a hemodialysis catheter directly in the RA by a cardiac surgery team. However, examples of this are not lacking. In a clinical case reported by E. Medinilla et al. the authors summarized that ideal vascular access should allow safe and prolonged access to the vascular system, have sufficient flow to conduct the planned HD, and be relatively unaffected by complications. The authors report a patient in whom all possibilities for conventional vascular access have been exhausted. On this occasion, they underwent implantation in the DP of a catheter for HD, as the access was a right anterolateral thoracotomy through the fifth intercostal space, and the catheter was led through a tunnel supraclavicular to the right. Ten months after placement, the catheter was functioning at an adequate flow rate, no postoperative complications have been identified. The conclusion is that the method is an adequate option in patients without any other alternative (2).

In a study published by Pereira M. et al, the implantation of a hemodialysis catheter by open cardiac surgery was called "life-saving". Data for this procedure in 7 patients for the period from 2004 to 2015 were presented. Patients included in this study had a history of multiple failed attempts to provide vascu- lar access to HD. The surgical techniques used were median sternotomy in the early years, minithoracotomy, and ministernotomy in the subsequent period. Early postoperative complications - bleeding and infections - have been reported in six out of seven and three out of seven, respectively, with two deaths, and late complications after discharge, the main one being the incidental change in the position of the HD catheter, which occurred in virtually all patients within 24 months of the procedure. Catheter thrombosis and catheter-related infections occurred in two out of seven patients. The conclusion they draw is that the placement of an intraatrial catheter for HD is associated with significant risk and high mortality. However, when the possibilities for alternative renal replacement therapy are exhausted, this approach must be considered (5).

Another study reported a catheter for HD implanted in the RA, with access to a median sternotomy, which continued to function properly 36 months after placement without serious complications. The authors suggest that intracardiac placement of a central venous catheter may be a safe and long-term option in cases of depletion of vascular access, which allows regular hemodialysis therapy (6).

Various surgical techniques for inserting a HD catheter directly into the RA have been described: median sternotomy, minithraracotomy, ministernotomy (5), right anterolateral thoracotomy (2), parasternal access $(7,8)$.

Such a procedure is performed for the first time in our department. We did not find data for such an approach in the Bulgarian sources. We recognize that the patient's condition must be tracked to assess the long-term outcome.

\section{CONCLUSION}

The use of open surgical access and the implantation of a hemodialysis catheter directly into the RA can be considered as a life-saving procedure in HD patients with acute uremic syndrome and exhausted vascular access.

\section{REFERENCES}

1. Gottschalk CW, Fellner SK. History of the science of dialysis. Am J Nephrol. 1997;17(3-4):289-98. doi: $10.1159 / 000169116$. 
2. Villagran Medinilla E, Carnero M, Silva JA, Rodriguez JE. Right intra-atrial catheter insertion at the end stage of peripheral vascular access for dialysis. Interact Cardiovasc Thorac Surg. 2011;12(4):648-9. doi: $10.1510 /$ icvts.2010.259879.

3. Chemla ES, Korrakuti L, Makanjuola D, Chang AR. Vascular access in hemodialysis patients with central venous obstruction or stenosis: one center's experience. Ann Vasc Surg. 2005;19(5):692-8. doi: 10.1510/icvts.2010.259879.

4. Vanholder R, Verbeke F, Van Biesen W. Dialysis: vascular access: not enough light at the end of the tunnel? Nat Rev Nephrol. 2009;5(10):551-2. doi: 10.1038/nrneph.2009.144.

5. Pereira M, Lopez N, Godinho I, Jorge S, Nogueira E, Neves F, et al. Life-saving vascular access in vascular capital exhaustion: single cen- ter experience in intra-atrial catheters for hemodialysis. J Bras Nefrol. 2017;39(1):36-41. doi: 10.5935/0101-2800.20170006.

6. Santos-Araujo C, Casanova J, Carvalho B, Pestana M. Prolonged use of an intracardiac catheter for dialysis in a patient with multiple venous access failure. Nephrol Dial Transplant. 2006;21(9):2670-1. doi: $10.1093 / \mathrm{ndt} / \mathrm{gfl} 154$.

7. Archundia AG, Mendoza AC, Manrique MN, Figueroa SA. A method to insert a haemodialysis catheter by parasternal access. Nephrol Dial Transplant. 2002;17(1):134-6. doi: 10.1093/ndt/17.1.134.

8. Yaşa H, Lafcı B, İlhan G, Tetik O, Özsöyler İ, Ergüne K, et al. Placing of permanent catheter through right anterior mini thoracotomy in patients with chronic renal failure. EJVES Extra. 2007;13(6):90-1. doi: 10.1016/j.ejvsextra.2007.03.001. 